\title{
Nanoimprinted plasmonic nanocavity arrays
}

\author{
Sangsik Kim, ${ }^{1}$ Yi Xuan, ${ }^{1}$ Vladimir P. Drachev,${ }^{2}$ Leo T. Varghese, ${ }^{1}$ Li \\ Fan, ${ }^{1}$ Minghao Qi, ${ }^{1}$ and Kevin J. Webb ${ }^{1, *}$ \\ ${ }^{1}$ School of Electrical and Computer Engineering and Birck Nanotechnology Center, Purdue \\ University, West Lafayette, IN 47907 USA \\ ${ }^{2}$ Department of Physics, University of North Texas, Denton, TX 76203, USA \\ *webb@purdue.edu
}

\begin{abstract}
We demonstrate high resonant absorption of visible light with a plasmonic nanocavity chain structure fabricated through resistless nanoimprinting in metal (RNIM). The RNIM approach provides a simple, reproducible, and accurate means to fabricate metallic nanopatterns with high fidelity. The nanocavities are shown to be efficiently excited using normally incident light, and the resonant wavelength can be controlled by either the width or the depth of the cavity. Numerical simulations confirm the experimental observations, and illustrate the behavior of the nanocavity chain waveguide and insensitivity to incident angle. The resonant absorption is due to the excitation of a localized metal-insulator-metal cavity mode. The interacting surface waves allow cavity lengths on the order of ten nanometers for light having a free space wavelength of about four hundred nanometers. Coupling of the cavities with an intervening surface plasmon wave results in a collective excitation and a chain waveguide mode that should prove valuable for more sensitive detection based on surface enhanced Raman scattering.
\end{abstract}

(C) 2013 Optical Society of America

OCIS codes: (220.4241) Nanostructure fabrication; (310.6628) Nanostructures; (260.2110) Electromagnetic optics; (350.4238) Nanophotonics and photonic crystals.

\section{References and links}

1. K. J. Webb and J. Li, "Analysis of transmission through small apertures in conducting films," Phys. Rev. B 73, 033401 (2006).

2. P. Genevet, J. P. Tetienne, E. Gatzogiannis, R. Blanchard, M. A. Kats, M. O. Scully, and F. Capasso, "Large enhancement of nonlinear optical phenomena by plasmonic nanocavity gratings," Nano Lett. 10, 4880-4883 (2010).

3. N. M. B. Perney, F. J. García de Abajo, J. J. Baumberg, A. Tang, M. C. Netti, M. D. B. Charlton, and M. E. Zoorob, "Tuning localized plasmon cavities for optimized surface-enhanced Raman scattering," Phys. Rev. B 76, 035426 (2007).

4. H. T. Miyazaki and Y. Kurokawa, "Controlled plasmon resonance in closed metal/insulator/metal nanocavities," Appl. Phys. Lett. 89, 211126 (2006).

5. Y. Kurokawa and H. T. Miyazaki, "Metal-insulator-metal plasmon nanocavities: Analysis of optical properties," Phys. Rev. B 75, 035411 (2007).

6. V. J. Sorger, R. F. Oulton, J. Yao, G. Bartal, and X. Zhang, "Plasmonic Fabry-Pérot nanocavity," Nano Lett. 9, 3489-3493 (2009).

7. A. Polyakov, S. Cabrini, S. Dhuey, B. Harteneck, P. J. Schuck, and H. A. Padmore, "Plasmonic light trapping in nanostructured metal surfaces," Appl. Phys. Lett. 98, 203104-203106 (2011).

8. A. Polyakov, H. A. Padmore, X. Liang, S. Dhuey, B. Harteneck, J. P. Schuck, and S. Cabrini, "Light trapping in plasmonic nanocavities on metal surfaces," J. Vac. Sci. Technol. B 29, 06FF01 (2011).

\#186800 - \$15.00 USD Received 11 Mar 2013; revised 25 Apr 2013; accepted 26 Apr 2013; published 17 Jun 2013

(C) 2013 OSA $\quad 1$ July 2013 | Vol. 21, No. 13 | DOI:10.1364/OE.21.015081 | OPTICS EXPRESS 15081 
9. S. Buzzi, F. Robin, V. Callegari, and J. F. Löffler, "Metal direct nanoimprinting for photonics," Microelectron. Eng. 85, 419-424 (2008).

10. S. Y. Chou, P. R. Krauss, and P. J. Renstrom, “25-nanometer resolution,” Science 272, 85-87 (1996).

11. S. H. Ko, I. Park, H. Pan, C. P. Grigoropoulos, A. P. Pisano, C. K. Luscombe, and J. M. J. Fréchet, "Direct nanoimprinting of metal nanoparticles for nanoscale electronics fabrication," Nano Lett. 7, 1869-1877 (2007).

12. A. T. Fafarman, S. H. Hong, H. Caglayan, X. Ye, B. T. Diroll, T. Paik, N. Engheta, C. B. Murray, and C. R. Kagan, "Chemically tailored dielectric-to-metal transition for the design of metamaterials from nanoimprinted colloidal nanocrystals," Nano Lett. 13, 350-357 (2013).

13. H. L. Chen, S. Y. Chuang, H. C. Cheng, C. H. Lin, and T. C. Chu, "Directly patterning metal films by nanoimprint lithography with low-temperature and low-pressure,” Microelectron. Eng. 83, 893-896 (2006).

14. L. T. Varghese, L. Fan, Y. Xuan, C. Tansarawiput, S. Kim, and M. Qi, "Resistless nanoimprinting in metal for plasmonic nanostructures," Small, http://dx.doi.org/10.1002/smll.201300168 (2013).

15. K. J. Webb and J. Li, "Resonant slot optical guiding in metallic nanoparticle chains," Phys. Rev. B 72, 201402 (2005).

16. E. D. Palik, Handbook of Optical Constants of Solids (Academic Press, 1998) vol. 3.

17. J. A. Scholl, A. L. Koh, and J. A. Dionne, "Quantum plasmon resonances of individual metallic nanoparticles," Nature 483, 421-427 (2012).

18. S. Shivanand, A. Ludwig, and K. J. Webb, "Impact of surface roughness on the effective dielectric constants and subwavelength image resolution of metal-insulator stack lenses," Opt. Lett. 37, 4317-4319 (2012).

19. K. J. Webb and J. Li, "Resonant waveguide field enhancement in dimers," Opt. Lett. 31, 3348-3350 (2006)

20. J. Homola, S. S. Yee, and G. Gauglitz, "Surface plasmon resonance sensors: review," Sensor Actuat B-Chem. 54, 3-15 (1999).

21. A. Dhawan, M. Canva, and T. Vo-Dinh, "Narrow groove plasmonic nano-gratings for surface plasmon resonance sensing," Opt. Express 19, 787-813 (2011).

22. H. Liu, Shivanand, and K. J. Webb, “Optical circuits from anisotropic films," Phys. Rev. B 79, 094203 (2009).

23. E. M. Purcell, "Spontaneous emission probabilities at radio frequencies," Phys. Rev. B 69, 681 (1946).

24. H. Altug, D. Englund, and J. Vučković, "Ultrafast photonic crystal nanocavity laser," Nat. Phys. 2, 484-488 (2006).

\section{Introduction}

Noble metals are good plasmonic materials, and they offer a complex dielectric constant at visible wavelengths with a relatively small imaginary component and a negative real part that provides for transverse magnetic surface waves. The collective oscillation of photons and electrons forms a surface plasmon-polariton wave, and coupling of two surfaces as a metal-insulatormetal (MIM) waveguide mode allows the squeezing of light down to the subwavelength length scale and large field enhancement in small cavities [1]. With visible light, cavity dimensions can be just a few tens of nanometers or less, and a footprint on the scale of current CMOS technology becomes possible. The field enhancement within a small mode volume makes the surface plasmon desirable in nonlinear optics [2] and for surface enhanced Raman scattering (SERS) [3]. More generally, plasmonic nanocavities provide sensing opportunities and the basis for nanolaser sources and for detectors, with the incorporation of appropriate materials into the cavities. The challenge is a suitable fabrication method to achieve adequate fabrication tolerances and suitable material arrangements for specific applications.

Several fabrication schemes have been used to realize plasmonic cavity structures [4-8]. A cavity formed with a MIM waveguide was demonstrated by depositing $\mathrm{Au} / \mathrm{SiO}_{2} / \mathrm{Au}$ films and using focused ion beam (FIB) milling to form a ridge $[4,5]$. However, use of a gap filling material (or support material, on the order of $10 \mathrm{~nm}$ of $\mathrm{SiO}_{2}$ in this case) restricts nonlinear optics and SERS applications. While FIB can be used to pattern metals by using a heavy ion to bombard and form features, it is a relatively time consuming process and has limited resolution. For nanostructures, electron beam lithography (EBL) is widely used for its high resolution patterning. Using EBL, a surface wave (metal - free space) cavity was achieved with two vertical Ag fins that act as mirrors [6]. Here, electroplated $\mathrm{Ag}$ was deposited over EBL patterned resist followed by lift-off. In this case, they achieved quality factor $\left(Q \approx \lambda_{0} / \Delta \lambda\right.$, where $\lambda_{0}$ is the free space resonant wavelength and $\Delta \lambda$ is the $3-\mathrm{dB}$ bandwidth of the resonance) ranging from 100 to

\#186800 - \$15.00 USD Received 11 Mar 2013; revised 25 Apr 2013; accepted 26 Apr 2013; published 17 Jun 2013 (C) 2013 OSA $\quad 1$ July 2013 | Vol. 21, No. 13 | DOI:10.1364/OE.21.015081| OPTICS EXPRESS 15082 
200. The dimension of the structure was dictated by the wavelength of the surface wave, which is close to the free space wavelength. Moreover, free space coupling remains a challenge. Recently, plasmonic light trapping in narrow nanogrooves on a metal surface was demonstrated by evaporating gold onto patterned hydrogen silsesquioxane (HSQ) resist lines [7, 8]. The patterned Au film was separated from the substrate either through wafer back-etching [7] or by peeling it off using epoxy [8]. Evaporating metal films over patterned resists often suffer from void formation due to a self shadowing effect. Also, it requires the complete back-etching of the primary substrate. In contrast, nanoimprint lithography can be used to pattern metal films with much ease and versatility.

It is well known that metal films can be shaped to the desired pattern by using ultra-high pressure and high temperature [9]. However, the use of extreme conditions is undesirable and not suitable for wafer-based processing. An alternative to this is nanoimprint lithography, where a mold is used to mechanically deform a soft material by applying low pressure and low temperature [10]. While this has normally been used to pattern polymer resists, it has also been used to directly pattern colloidal Au nanoparticles, allowing feature sizes down to about $100 \mathrm{~nm}$ to be achieved $[11,12]$. This method provided a convenient fabrication approach to pattern various wire and dot structures for resistors and transistors [11], and for metamaterials [12]. While colloidal metal nanoparticles can be easily formed into shapes, metal films are difficult to be patterned, owing to their higher elastic modulus and hardness. To counter this, a soft intermediate layer has been added between the substrate and the metal film to realize metal patterns with gentle contours using low pressure and low temperature [13]. However, many high performance plasmonic devices require high quality noble metal surfaces with precise features. Hence, it is important to use a low pressure and low temperature process that forms patterns on high quality metal films without any intermediate layers.

In this work, we demonstrate a plasmonic nanocavity chain structure using resistless nanoimprinting in metal (RNIM) [14]. A Si mold with sparse nanoscale patterns is used to directly imprint $\mathrm{Ag}$ films in a single step using low pressure/temperature to fabricate devices with high throughput and low-cost. With etching techniques for Si being well developed, RNIM allows nanoscale resolution and sharp features in $\mathrm{Si}$ to be realized on metal through its high fidelity pattern transfer. The experimental results for the nanocavity chain structures fabricated by RNIM are supported by numerical simulations. The structure shows polarization sensitive and angular insensitive absorption, and has high field enhancement within the nanometer-scale cavity dimensionsshows the RNIM process flow.

\section{Device fabrication}

Figure 1 shows the RNIM process flow. The mold was fabricated on a Si wafer by using EBL $(100 \mathrm{kV})$. HSQ, a negative EBL resist, was spun to a thickness of $50 \mathrm{~nm}$ on a Si wafer, exposed and developed. The HSQ patterns were transferred to Si using reactive ion etching (RIE) with $\mathrm{Cl}_{2} / \mathrm{Ar}$ chemistry, and HSQ was removed with a brief dip in HF acid to form the mold. A 5 $\mathrm{nm} \mathrm{Cr}$ layer was deposited on a quartz substrate to promote adhesion, and then a $\mathrm{Ag}$ film was deposited with an electron beam evaporator. Before nanoimprinting, the mold was cleaned by a standard RCA process, while the metal film was sonicated in a solvent. RNIM was performed on a Nanonex NX 2000, a commercial resist-based nanoimprint lithography tool, by applying a pressure of $4 \mathrm{MPa}$ and with temperatures between 100 and $150{ }^{\circ} \mathrm{C}$. After nanoimprinting, the mold and substrate were manually separated.

Figure 2(a) shows a Si mold and the resulting imprint on a $200 \mathrm{~nm}$ thick Ag film is shown in Fig. 2(b). The grating and grid patterns of the mold pressed into the Ag film result in square dot arrays and ridges. The Si gratings shown in Fig. 2(c) were imprinted into a $\mathrm{Ag}$ film, resulting in the Ag gratings shown in Fig. 2(d). The sharp edges of the imprinted metal pattern shows the

\#186800 - \$15.00 USD Received 11 Mar 2013; revised 25 Apr 2013; accepted 26 Apr 2013; published 17 Jun 2013 (C) 2013 OSA $\quad 1$ July 2013 | Vol. 21, No. 13 | DOI:10.1364/OE.21.015081 | OPTICS EXPRESS 15083 


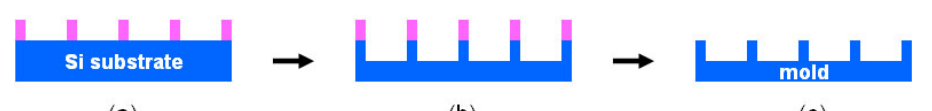

(a)

(b)

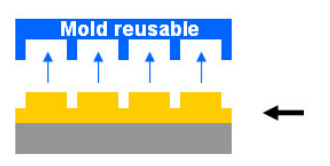

(f)

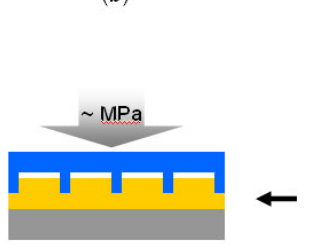

(e) (c)

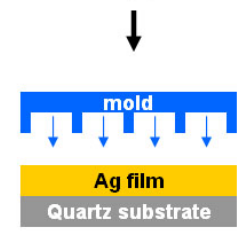

(d)

Fig. 1. The RNIM process: (a) patterning of HSQ resist; (b) Si dry etching with $\mathrm{Cl}_{2} / \mathrm{Ar}$ chemistry; (c) removal of HSQ by HF to form the mold; (d) contact of mold to the metal film; (e) nanoimprinting; (f) removal of the Si mold.

(a)

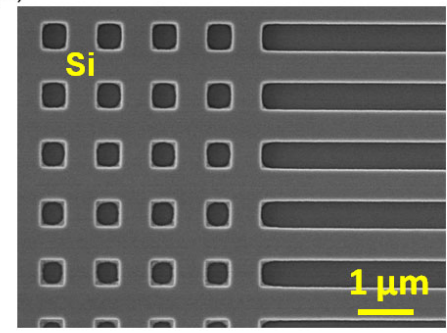

(c)

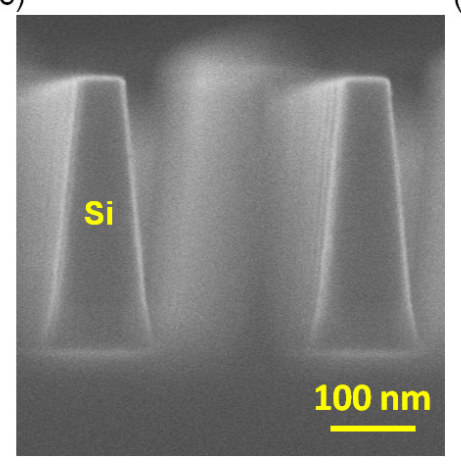

(b)

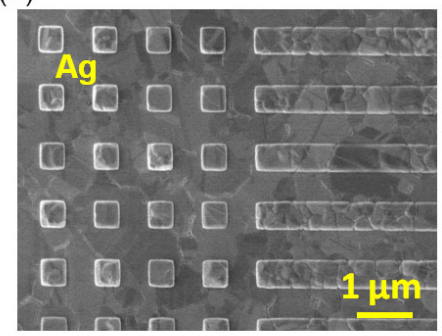

(d)

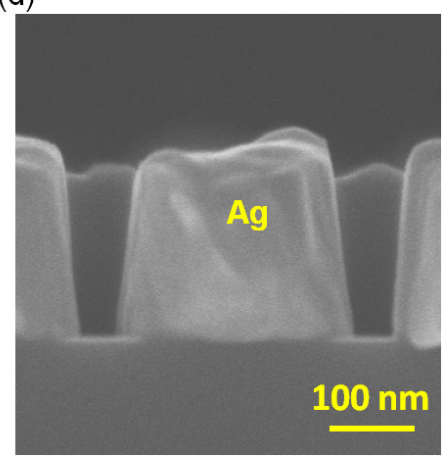

Fig. 2. SEM results of the RNIM process. (a) A Si mold with gratings and grids. (b) Imprinted Ag film using the mold in (a). (c) Cross sectional view of a Si mold. (d) Cross sectional view of the imprinted $\mathrm{Ag}$ film with the mold in (c).

high-fidelity pattern transfer of the RNIM process. The residual metal inside the trenches can be kept to a minimum by using molds with larger depths than the metal thickness and ensuring the mold patterns are sparsely populated to allow localized pressures around the patterns to be much higher than the metal's yield strength [14].

We designed the nanocaivty array by choosing a slot width we could achieve $(25 \mathrm{~nm})$ with a reasonable quality. The grating period, $P$, was fixed to $100 \mathrm{~nm}$. A two-dimensional (2D) finite element method (FEM) model (COMSOL Multiphysics) was used to design for a particular resonant wavelength (400 $\mathrm{nm}$ in this case) by varying the depth of the cavity. Details of the 


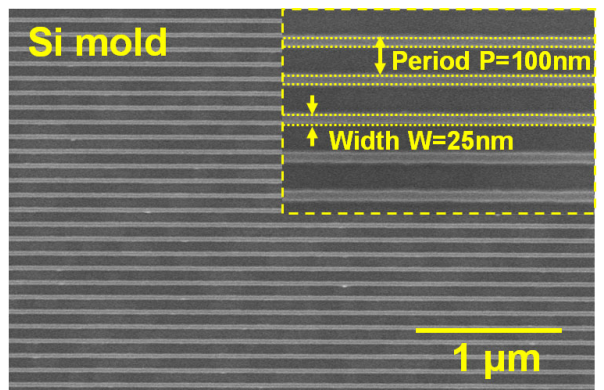

(a) Si mold

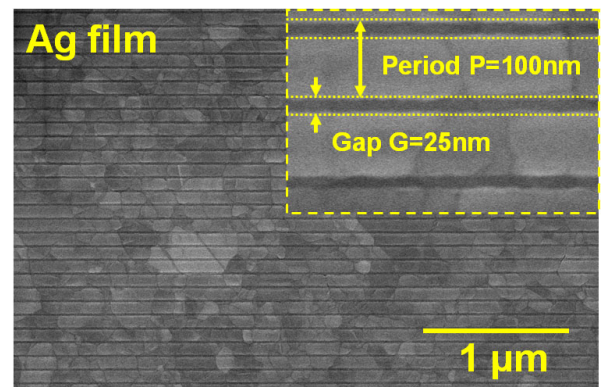

(b) Ag film

Fig. 3. SEM images of the nanocavity array. (a) The Si mold used to generate the plasmonic nanocavity array. (b) The imprinted Ag film with trenches matching the mold dimensions shown in (a).

simulations are given in Section 4. In the fabrication, the depth of each cavity was adjusted by using different $\mathrm{Si}$ mold heights. The nanocavity array was fabricated by patterning Si gratings over an area of $20 \mu \mathrm{m} \times 25 \mu \mathrm{m}$ with $25 \mathrm{~nm}$ wide lines and a $100 \mathrm{~nm}$ period, as shown in Fig. 3(a). The resulting imprint on a $200 \mathrm{~nm}$ thick Ag film is shown in Fig. 3(b).

\section{Experimental setup and measurement}

To measure the reflectance of the plasmonic nanocavity chain, a custom built experiment that included an Acton Research spectrophotometer was used. Figure 4(a) shows the experimental setup. A white light source (Fiber-Lite Illuminator series 180) provided normal illumination through a polarizer, allowing us to make measurements with transverse magnetic (TM) or transverse electric (TE) wave excitation. The polarized beam was directed into the objective lens through the partially reflecting (50-50) beam splitter, and either a 20x or 50x objective lens was used to focus the light onto the sample. The reflected light was collected by the same lens and detected by a spectrometer with a CCD detector through the beam splitter. By careful alignment, an area less than the device size was spectrally resolved. The illumination spot size was about $50 \mu \mathrm{m}$ and roughly a $5 \mu \mathrm{m}$ spot was imaged onto the spectrometer. The alignment was achieved using a white light image on a CCD camera, within which the spectrally resolved point and region was known.

In order to observe and compare the performance of the plasmonic nanocavity chain structure, four intensity measurements were made on each device, as shown in Fig. 4(b): TE illumination on the sample $\left(I_{\mathrm{TE}, \mathrm{on}}\right)$, TE illumination off the sample $\left(I_{\mathrm{TE}, \text { off }}\right)$, TM illumination off the sample $\left(I_{\mathrm{TM}, \text { off }}\right)$, and TM illumination on the sample $\left(I_{\mathrm{TM}, \mathrm{on}}\right)$. The localized cavity mode can be excited only with TM light $\left(I_{\mathrm{TM}, \text { on }}\right)[1,15]$. The other measurements were performed for calibration. To observe the performance of the devices, the power absorption ratios of the optical cavities were compared by measuring the relative reflectance $I_{T M, o n} / I_{T M, o f f}$. For our process, we believe that the top metal surface in the slot region is roughened because of a pile-up of the metal displaced from the slots [14]. The resulting roughness difference modifies the reflectance on and off the device. This scatter is accounted for by using TE measurements. Thus, we formed calibrated, relative reflectance data as

$$
R_{\mathrm{r}, \exp }=\frac{I_{\mathrm{TM}, \mathrm{on}}}{I_{\mathrm{TM}, \mathrm{off}}} \cdot \frac{I_{\mathrm{TE}, \mathrm{off}}}{I_{\mathrm{TE}, \mathrm{on}}},
$$

where $I_{\mathrm{TM}, \mathrm{on}}, I_{\mathrm{TM}, \text { off }}, I_{\mathrm{TE}, \mathrm{on}}$, and $I_{\mathrm{TE}, \text { off }}$ are the measured spectral data. 


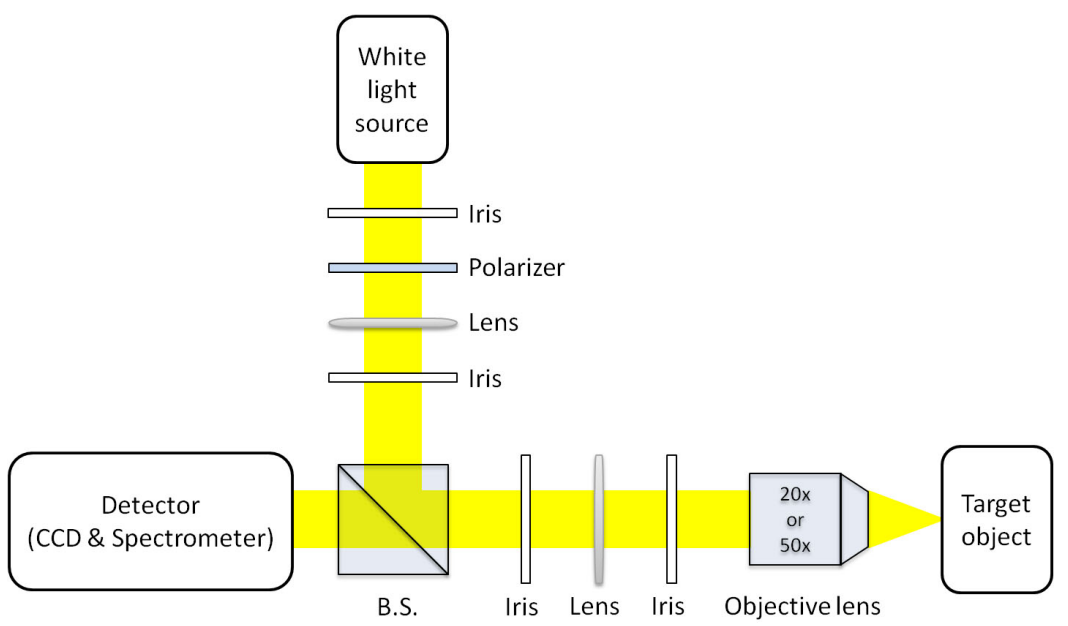

(a) Experimental setup

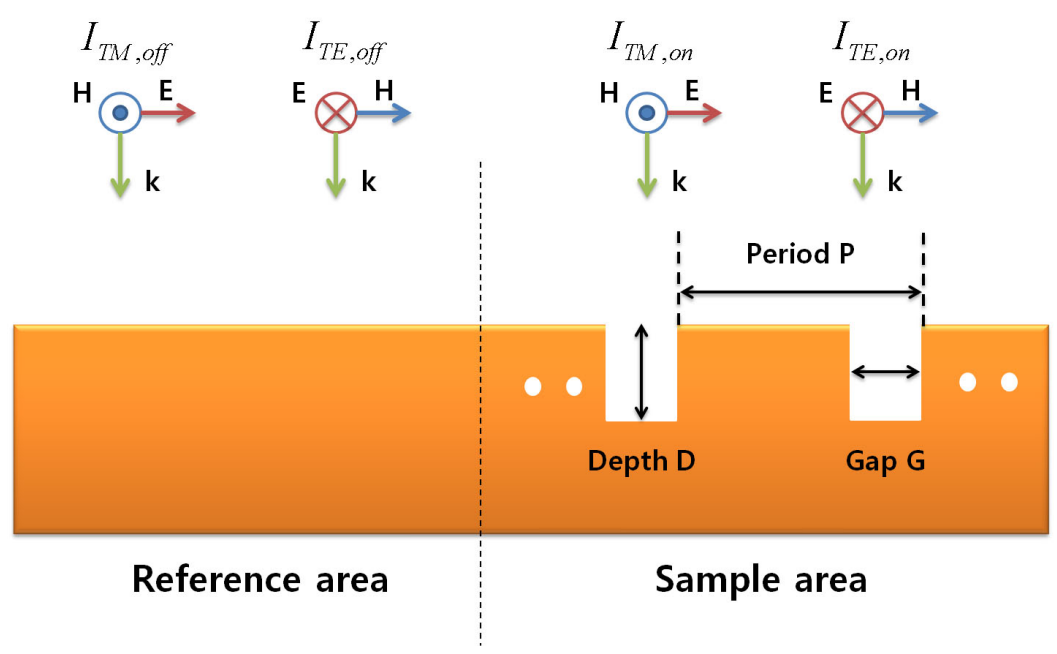

(b) Sample dimension and four measured signals

Fig. 4. (a) Schematic for the reflectance spectrometer measurement. (b) Nanocavity chain dimensions and the four measured intensities: TM illumination on the sample $\left(I_{\mathrm{TM}, \text { on }}\right)$ and off the sample $\left(I_{\mathrm{TM}, \text { off }}\right)$, and TE illumination on the sample $\left(I_{\mathrm{TE}, \mathrm{on}}\right)$ and off the sample ( $I_{\mathrm{TE}, \text { off }}$ ), all used to form the calibrated reflection $R_{\mathrm{r}, \exp }$.

\section{Results and discussion}

Figure 5(a) shows the measured data corresponding to (1) for devices with various cavity depths $(D)$ and a fixed gap $(G=25 \mathrm{~nm})$ and period $(P=100 \mathrm{~nm})$. Numerical simulations used a 2D FEM model (COMSOL Multiphysics). The simulation domain had 10 nanocavities, with a periodic boundary condition imposed on each end of the structure. Perfectly matched layers (PMLs) were placed on both the top and bottom of the domain. For comparison, the reflected power ratio of (1), as used for the experimental data, was numerically calculated. The numerical reflectance simulations, $R_{\mathrm{r}, \text { sim }}$, are plotted in Fig. 5(b) for the nanocavity dimensions used in the experiment and with varying $D$. Notice that the resonance wavelengths in the experimental 


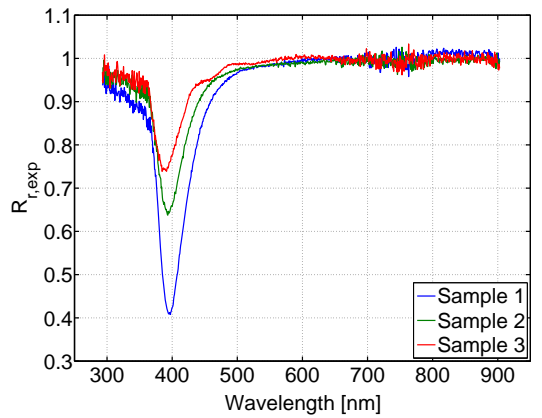

(a) Experiment

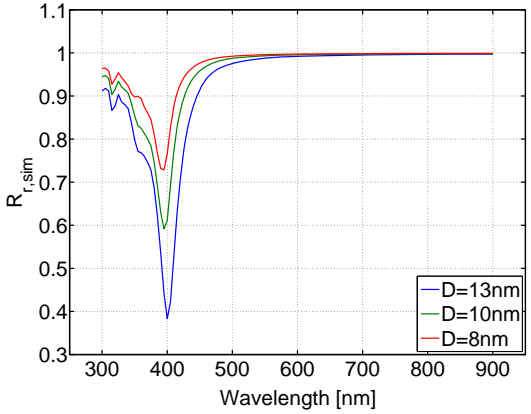

(b) Simulation

Fig. 5. (a) Measured and (b) simulated relative reflectivity of the plasmonic nanocavities. Referring to Fig. 4(b), the cavity gap size is $G=25 \mathrm{~nm}$ and the grating period is $P=$ $100 \mathrm{~nm}$. The different lines are for the various cavity depths $D: 13 \mathrm{~nm}$ (blue), $10 \mathrm{~nm}$ (green), and $8 \mathrm{~nm}$ (red).

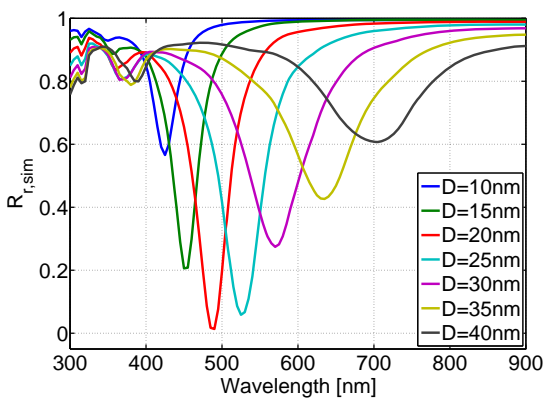

Fig. 6. Simulated relative reflectivity for the plasmonic nanocavity array for normal incidence with gap size $G=10 \mathrm{~nm}$, grating period $P=100 \mathrm{~nm}$, and depths $D$ of: $10 \mathrm{~nm}$ (blue), $15 \mathrm{~nm}$ (green), $20 \mathrm{~nm}$ (red), $25 \mathrm{~nm}$ (aqua), $30 \mathrm{~nm}$ (pink), $35 \mathrm{~nm}$ (yellow), and $40 \mathrm{~nm}$ (black).

data in Fig. 5(a) are almost identical to those in the simulated results of Fig. 5(b), except for slight differences in the resonance dips. Predicting the correct resonant wavelengths requires correct $G, D$ and metal complex dielectric constant parameters. We used published measured bulk data for the complex dielectric constant for Ag [16]. There have been several reports of the differences between the effective optical parameters and the bulk material properties when the structure size approaches the atomic scale or surface roughness increases [17, 18]. Our effectiveness in capturing the measured data with the simulations suggests these issues are not important here, and may attest to the quality of the cavities (or at least that surface roughness is not a problem). The measured and simulated quality factors are virtually the same, and are dictated by loaded (cavity coupling) and intrinsic (dissipative) contributions.

The resonant dips in the reflected light are caused by the excitation of the localized cavity mode and may involve inter-cavity coupling via a propagating surface wave. The plasmonic nanocavities, with relevant dimensions that are much smaller than the free space optical wavelength $\left(G \ll \lambda_{0}\right)$ were designed to resonate at around $\lambda_{0}=400 \mathrm{~nm}$, and the light at this wavelength is confined inside the cavities at resonance, resulting in the dips in the reflected power shown in Fig. 5. Notice that only $40 \%$ of the normally incident light is reflected on resonance for the $D=13 \mathrm{~nm}$ case. For $D=13 \mathrm{~nm}$, the $Q$ is roughly 10 , and the smaller $D$ cavities have 


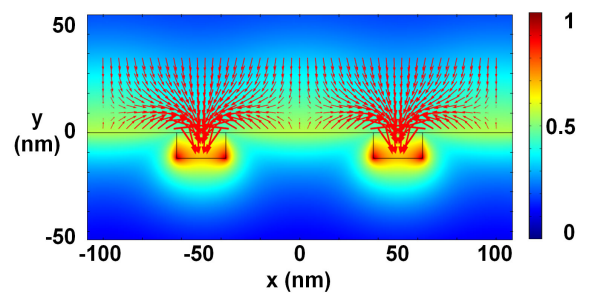

(a) Color contours: normalized $\left|H_{z}\right|$, Arrows: Poynting vector

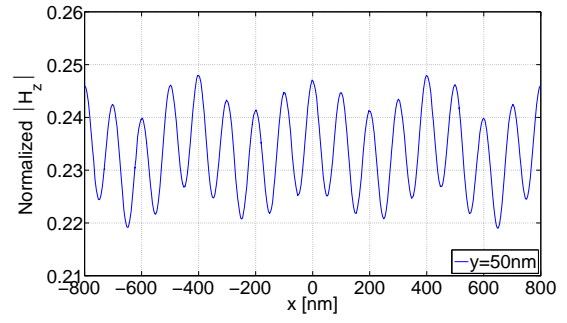

(b) Normalized $\left|H_{z}(x)\right|$ at $y=50 \mathrm{~nm}$

Fig. 7. (a) The normalized simulated $\left|H_{z}(x, y)\right|$, plotted in the neighborhood of two cavities, is shown as color contours. The spatially-dependent Poynting vector is shown by the red arrows (at the point where the arrow begins and with relative magnitude given by the arrow length). (b) Plot of the normalized $\left|H_{z}(x, y=50 \mathrm{~nm})\right|, 50 \mathrm{~nm}$ above the metal surface, with parameters: $D=13 \mathrm{~nm}, G=25 \mathrm{~nm}$, and $P=100 \mathrm{~nm}$. In both (a) and (b), the free space wavelength is $\lambda_{0}=400 \mathrm{~nm}$.

slightly lower $Q$ for reasons that relate to geometry and material dispersion. Also, Fig. 5 shows how the cavity resonance shifts as the sample depth varies. For a fixed cavity gap size, $G$, the same MIM waveguide mode is excited, but this mode has a resonant wavelength that depends on cavity depth. This dependence on $D$ is more noticeable as $G$ reduces. Figure 6 shows simulated results with a gap size of $G=10 \mathrm{~nm}$. While a larger $D$ increases the resonant wavelength in Fig. 6, the $Q$ is maximum for the intermediate wavelength range shown $(D=20 \mathrm{~nm}$, the red curve).

The normalized simulated magnetic field $\left(\left|H_{z}\right|\right)$ is shown as a color contour map in Fig. 7(a) for $G=25 \mathrm{~nm}$ and $D=13 \mathrm{~nm}\left(P=100 \mathrm{~nm}\right.$, with normal TM excitation at $\left.\lambda_{0}=400 \mathrm{~nm}\right)$. The power flow, represented by the Poynting vector, is indicated by the red arrows. Figure 7(a) shows the large power density directed into the cavities, where the fields are highest. Note that we show only the Poynting vector above the metal surface and in free space $(y>0)$. When TM light illuminates the structure, surface charges on the two opposite metal walls allow the narrow gap to excite the MIM waveguide mode [19], and this coupled surface waveguide mode resonates within the cavity, reflecting from the top (waveguide to free space interface) and bottom of the cavity. Figure 7(b) shows a plot of the normalized $\left|H_{z}(x)\right| 50 \mathrm{~nm}$ above the surface. We can observe the standing wave periodic envelope that corresponds to the wavelength of the propagating plasmon mode along the top surface. This is the chain waveguide mode that couples the cavities and is equivalent to the nanoparticle chain waveguide operation with resonant gaps [15]. We therefore identify the periodic envelope as the existence of the surface wave that is excited with normal light. The rapid field variation in Fig. 7(b) is associated with the large magnetic field at the metal surface between the cavities and the small field at the entrance to each cavity, i.e., the distance between the maximum or minimum magnetic field points in Fig. 7(b) is $P$, the grating period. The surface wave in Fig. 7(b) occurs with normally incident light because the phase match condition is satisfied by the cavity periodicity.

The localized cavity mode resonance dips in the reflected light differ from those in typical propagating surface plasmon sensors that use either a prism or grating coupler to excite the propagating surface wave [20]. Propagating surface wave sensors are restricted to a specific illumination angle to satisfy the phase matching condition. The reflection dip in those sensors occurs with coupling into the surface wave, and this coupling sensitivity is the basis for sensing, i.e., introduction of a material on the surface with a different refractive index changes the coupling condition, moving the resonant wavelength and providing a sensing mechanism [20]. 


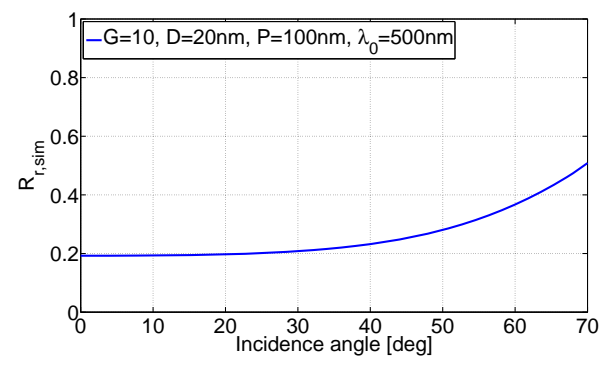

(a)

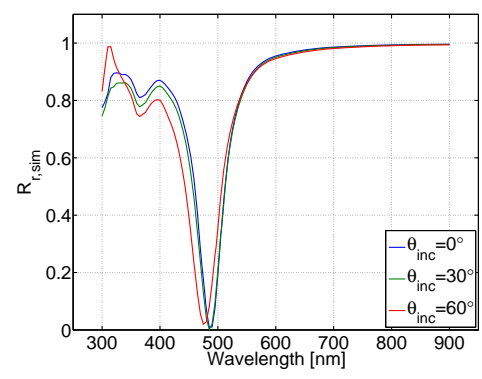

(b)

Fig. 8. (a) Simulated relative reflectivity for the plasmonic nanocavity array for TM light at $\lambda_{0}=500 \mathrm{~nm}$ as a function of incidence angle $\left(\theta_{\text {inc }}\right)$ with a gap size $G=10 \mathrm{~nm}$, a grating period $P=100 \mathrm{~nm}$, and a depth $D=20 \mathrm{~nm}$. (b) The same calculation as (a), but as a function of excitation wavelength for different illumination angles: $\theta_{\text {inc }}=0^{\circ}$ (blue), $\theta_{\text {inc }}=30^{\circ}$ (green), and $\theta_{\text {inc }}=60^{\circ}$ (red).

Our plasmonic nanocavity chain structure produces a resonance dip in the reflected power by exciting the localized cavity mode, and the resonant wavelength and width is dictated by the cavity resonance. In an application, the nanocavity array can be excited with normally incident light and without the need for a phase matching prism or grating, as has been noted from prior numerical studies [21].

Figure 8 (a) shows the simulated relative reflectance, $R_{\mathrm{r}, \text { sim }}$, as a function of TM illumination angle $\left(\theta_{\text {inc }}\right)$. The cavity array geometry was set to $G=10 \mathrm{~nm}, D=20 \mathrm{~nm}$, and $P=100 \mathrm{~nm}$, and the excitation was at $\lambda_{0}=500 \mathrm{~nm}$. Notice that the reflectance is quite insensitive to illumination angle. Earlier work used various metal-insulator stack metamaterials to reduce the angular dependence of the reflected light [22]. Here, this is achieved because of the influence of the cavity array and occurs for wavelengths in the neighborhood of the cavity resonance. In Fig. 8 (b), the calculated $R_{\mathrm{r} \text {,sim }}$ is shown as a function of wavelength for different illumination angles $\left(\theta_{\text {inc }}\right)$. Notice that the resonant wavelength changes little with varying incidence angle. The angular insensitivity in exciting the localized cavity mode will make it easier to incorporate the nanocavity array with other measurement systems and to miniaturize the sensor [21].

\section{Conclusion}

While we presented results for RNIM-fabricated plasmonic nanocavity chains having a gap size of $G=25 \mathrm{~nm}$, a period $P=100 \mathrm{~nm}$, and depth $(D)$ from $8-13 \mathrm{~nm}$, substantially smaller $G$ and larger $D / G$ aspect ratios should be possible, and of course $P$ can be easily varied. The RNIM method provides a simple fabrication approach to achieve nanometer-scale precision in a family of plasmonic waveguides and devices. The ability to control the resonance characteristics through cavity design and insensitivity to incidence angle should prove important in sensing applications. The large surface and cavity fields suggest application in SERS-based sensors and for nonlinear optical devices. Also, small mode volume plasmonic nanocavities have the potential to achieve nanolasers with a high Purcell factor [23,24].

\section{Acknowledgments}

This work was supported by the National Science Foundation (Grant 0901383). VPD acknowledges partial support from the Air Force Research Laboratory Materials and Manufacturing Directorate Applied Metamaterials Program.

\#186800 - \$15.00 USD Received 11 Mar 2013; revised 25 Apr 2013; accepted 26 Apr 2013; published 17 Jun 2013

(C) 2013 OSA

1 July 2013 | Vol. 21, No. 13 | DOI:10.1364/OE.21.015081 | OPTICS EXPRESS 15089 